\title{
The role of palbociclib in thyroid carcinoma with BRAF mutation
}

\author{
Nikolaos Tsoukalas ${ }^{1,2 \#}$, Konstantinos Tsapakidis ${ }^{2 \#}$, Krystallenia I. Alexandraki ${ }^{3}$ \\ ${ }^{1}$ Department of Oncology, Veterans Hospital (NIMTS), Athens, Greece; ${ }^{2}$ Department of Oncology, Faculty of Medicine, School of Health Sciences, \\ University of Thessaly, Larissa, Greece; ${ }^{3}$ Endocrine Unit, 1st Department of Propaedeutic Medicine, Laiko University Hospital, Medical School, \\ National and Kapodistrian University of Athens, Athens, Greece \\ \#These authors contributed equally to this work. \\ Correspondence to: Nikolaos Tsoukalas, MD, MSc, PhD. Department of Oncology, Veterans Hospital (NIMTS), Gennimata N. 10-12 Ampelokipi, \\ 11524 Athens, Greece. Email: tsoukn@yahoo.gr. \\ Provenance: This is a Guest Editorial commissioned by Section Editor De-Tao Yin (Department of Thyroid Surgery, the First Affiliated Hospital of \\ Zhengzhou University, Zhengzhou, China). \\ Comment on: Antonello ZA, Hsu N, Bhasin M, et al. Vemurafenib-resistance via de novo RBM genes mutations and chromosome 5 aberrations is \\ overcome by combined therapy with palbociclib in thyroid carcinoma with BRAFV600E. Oncotarget 2017;8:84743-60.
}

Submitted Mar 04, 2018. Accepted for publication Mar 14, 2018.

doi: $10.21037 /$ gs.2018.03.07

View this article at: http://dx.doi.org/10.21037/gs.2018.03.07

Recurrent iodine-refractory differentiated thyroid cancer (RIR-DTC) is the centre of recent investigation on endocrine oncology. The treatment of most patients with differentiated thyroid cancer (DTC) includes surgery, thyroid hormone therapy, and selective use of radioactive iodine. However, for those patients with metastatic DTC that progresses despite radioiodine, TSH-suppressive thyroid hormone therapy, and external beam radiotherapy (EBRT), treatment options have historically been limited. New approaches based upon application of targeted chemotherapies are emerging as effective alternatives for progressive disease. For patients with metastatic DTC that persists, treatment options include observation, kinase inhibitors that primarily target angiogenesis, and traditional cytotoxic chemotherapy. The most recent American Thyroid Association (ATA) guideline (1) states for the first-line licenced medical treatment of RIR-DTC, the tyrosine kinase inhibitor (TKI) therapy that is eligible for patients with "imminently threatening disease progression expected to require intervention and/or to produce morbidity or mortality in $<6$ months, or symptomatic disease". However, medical therapy, particularly these newer "targeted therapies" have significant toxicities and cost, therefore, it is important to limit the use of systemic treatments to patients at significant risk for morbidity or mortality due to progressive metastatic disease (2). Conventional cytotoxic agents and TKIs are occasionally used for the treatment of patients with progressive symptomatic thyroid cancer that is unresponsive or not amenable to surgery, radioiodine therapy, or external radiotherapy. The National Comprehensive Cancer Network (NCCN) Guidelines also consider TKI therapies for progressive and/or symptomatic RIR-DTC but not in cases of central nervous system metastases (3). Only a phase II and phase III study of lenvatinib and sorafenib, respectively, define the ideal timing for the start of the first licenced medical therapy, namely TKI therapy, by the progression in the past year $(4,5)$. Other TKIs have also been tried in DTC. Cabozantinib, licenced for medullary thyroid carcinoma, has been tried in a phase II study in RIRDTC and evidence of disease progression on prior VEGFtargeted therapy (6). Pazopanib has been given under an individualized scheme measuring drug levels and comparing continuous $v s$. intermittent pazopanib administration (Guidelines 2015: NCT01552356; NCT01813136).

The basis of medical treatment in thyroid cancer consist on the identification of gene mutations and rearrangements, as follicular thyroid cancers have RAS mutations with the NRAS mutations being more common than HRAS and KRAS, and papillary thyroid cancers frequently carry gene mutations and rearrangements that lead to activation of the mitogen-activated protein kinase (MAPK) that promotes cell division. Rearrangements of RET and NTRK1 tyrosine kinases, activating mutations of BRAF, 
and activating mutations of RAS are sequential components leading to activation of MAPK. Finally, some anaplastic and poorly DTCs contain the same BRAF or RAS mutations as the regions of anaplastic thyroid cancer $(7,8)$. Based upon evidence of overexpression of vascular endothelial growth factor (VEGF) and its receptors (VEGFRs), it becomes clear that targeting angiogenesis has resulted in the most impressive clinical responses to date in DTC $(9,10)$. Sorafenib is an oral, small molecule multikinase inhibitor (MKI) targeting VEGFR 1, 2, and 3, platelet-derived growth factor receptor (PDGFR), common RET/PTC subtypes, c-kit, and less potently, BRAF. It is approved for the treatment of locally recurrent or metastatic, progressive DTC, irrespective of histology and molecular subtype, that no longer responds to radioactive iodine treatment. Lenvatinib is another oral, MKI targeting VEGFR1-3, fibroblast growth factor receptor (FGFR)1-4, PDGFRa, rearranged during transfection (RET), and c-KIT (5). Cabozantinib is an oral small-molecule multi-targeted TKI with potent activity against VEGFRs and $\mathrm{N}$-methyl-N1-nitroso-guanidine human osteosarcoma transforming gene (MET), displaying also activity against the RET gene, the growth arrest-specific 6 (GAS6) gene receptor (AXL), and the c-KIT, TIE2, and fms-like tyrosine kinase (FLT) 3 genes $(11,12)$.

Additional molecular pathways seem to be implicating in pathophysiology of DTC. The activation of MAP kinase pathway can increase iodine uptake in radioiodinerefractory DTC because it regulates a number of genes involved in iodine metabolism. So, by using kinase inhibitors targeting MAPK it restores expression of iodine metabolizing proteins and increases susceptibility to RAI as demonstrated with the MEK inhibitor selumetinib driving to tumor shrinkage (13) with similar results seen with the BRAF inhibitor dabrafenib (14). Small molecule inhibitors targeting signaling kinases have been of interest for the treatment of advanced DTC, given the oncogenic roles of mutations in the serine kinase BRAF, and tyrosine kinases RET (in the mutated fusion protein RET/PTC, the "RET/PTC oncogene") and RAS, and the contributory roles of tyrosine kinases in growth factor receptors such as the VEGFR (15). The MAPK pathway it is being activated by the RAF proteins and mainly the T1799A mutation of the BRAF gene, a protein found in more than $50 \%$ of malignant melanomas, $10 \%$ of colon cancers and $29-69 \%$ of papillary thyroid cancer (16). BRAF mutations include point mutations, deletions, insertions or chromosomal rearrangement and may confer a poor clinical prognosis, because recurrences occur more often and BRAF mutations may confer a worse clinical prognosis, as we can see them in patients with advanced stage, lymph node metastases and extrathyroidal invasion; nevertheless, for these findings, conventional histologic evaluation was as effective in predicting outcome as was the presence of the BRAF mutation $(17,18)$. As opposed to these findings, in international, randomized phase III trials of sorafenib and lenvatinib (as mentioned above), patients in the placebo arm experienced similar progression-free survival (PFS) irrespectively of the BRAF V600E mutation. The most usual mutation, about $98-99 \%$, is a replacement of valine to glutamate at codon 600 , by changing the nucleotide thymine at position 1,799 to adenine (19). Thus, when a patient with advanced disease has BRAF mutation, this finding does not change the prognosis, but the use of BRAF V600E inhibitor for aggressive DTC has been questioned (13).

Seconds mutations blocking the binding site of the specific drug to BRAF have not been found in DTC, as in other malignancies. Several resistance mechanisms have been described that typically involve tumor cell reactivation via the MAPK pathway through a variety of alternative means (20). Cells with mutations of BRAF V600E were used to help understand acquired resistance to BRAF inhibitors, how BRAF mutated cells survive BRAF inhibition. Bypass mechanisms within the MAPK pathway can restore ERK activation, regardless of ongoing BRAF inhibition. ERK stimulation induce pro-growth and transforming factors, through its interaction with a number of molecules, which appears to be critical for the development of many cancers (21). Restoration can be accomplished by several mechanisms, like increasing the number of several tyrosine kinases (i.e., PDGFRB, ERBB2), activation of NRAS via mutation, upregulation of CRAF, activating mutations of MEK, activation of the serine/threonine MAPK kinases (COT), overexpression of mutant BRAF, and loss of NF1 (20,22-25). Shortened forms of the BRAF protein may be produced due to altered RNA processing. These modified forms of the BRAF protein can activate the MAPK pathway even in the presence of a BRAF inhibitor (26). Signaling through the parallel growth and survival PI3K pathway, by insulin growth factor receptor 1 (IGF-1R) expression or AKT1 mutation, is another way of acquired resistance that has been described and fits the definition of an outside-of-pathway bypass mechanism (23).

The ERK pathway plays a crucial role by activating several genes inducing cell cycle entry and suppressing negative regulators of the cell cycle. An example is cyclin D1-Cdk4 and -Cdk6 complexes (Cdk4/6) a phosphorylation 
held by ERK after nuclear localisation (27). Preclinical studies suggest that elevated pretreatment levels of CCND1 amplification in tumors, is crucial because it leads to the overexpression of cyclin D1 and elevated CDK4 expression, so it maybe a as biomarker and we need to further investigate (28). In evaluation of baseline samples obtained from patients with BRAF-mutant melanoma prior to treatment with dabrafenib, loss of the cyclin dependent kinase inhibitor CDKN2A (p16INK4a) and amplification of CCND1 were associated with poorer outcome to therapy (29). The current article confirmed the use of palbociclib to tumor cells, either with primary or secondary resistance to vemurafenib (30). Palbociclib is a highly selective, reversible inhibitor of CDK4 and 6 and is already being used for hormone receptor positive metastatic breast cancer $(31,32)$. This combined therapy resulted in a very effective strategy to target tumor cells with either resistance. So, combination therapy strategies could overcome this intrinsic resistance by co-targeting of CDK4/6 and BRAF and clinical combination studies are needed to validate this hypothesis.

The recent guidelines underlines that many patients with metastatic disease do not progress rapidly, so postpone the medical treatment from patients with stable, asymptomatic metastatic disease to patients with progressive disease at risk to become symptomatic is reasonable. Whatever TKI is used as initial therapy, many patients will eventually receive several TKIs, and nowadays other inhibitors of small molecules impacting over their disease natural history. The main drawback of all these drugs is the adverse effects that are not always easily manageable rendering these therapeutic modalities less preferable from the patients who prefer a better quality of life $(4,5,33)$. However, the present study implies that as in other types of cancer it is not only the patient factor that till now could affect the decision making of starting the one drug over the other but the tumor factor as well. These obstacles are overcoming by the combination of several treatments. It remains to be elucidated the exact hierarchy of lines of treatments since we have many treatments, like radioiodine resensitization therapy, immunotherapy, or drugs against specific molecular targets as tyrosine or MEK kinases and checkpoint factors has come.

\section{Acknowledgements}

None.

\section{Footnote}

Conflicts of Interest: The authors have no conflicts of interest to declare.

\section{References}

1. Haugen BR, Alexander EK, Bible KC, et al. 2015 American Thyroid Association Management Guidelines for Adult Patients with Thyroid Nodules and Differentiated Thyroid Cancer: The American Thyroid Association Guidelines Task Force on Thyroid Nodules and Differentiated Thyroid Cancer. Thyroid 2016;26:1-133.

2. Ito $\mathrm{Y}$, Suzuki S, Ito K, et al. Tyrosine-kinase inhibitors to treat radioiodine-refracted, metastatic, or recurred and progressive differentiated thyroid carcinoma [Review]. Endocr J 2016;63:597-602.

3. NCCN clinical practice guidelines in oncology, thyroid carcinoma 2017 Version 2.

4. Brose MS, Nutting CM, Jarzab B, et al. Sorafenib in radioactive iodine-refractory, locally advanced or metastatic differentiated thyroid cancer: a randomised, double-blind, phase 3 trial. Lancet 2014;384:319-28.

5. Schlumberger $M$, Tahara $M$, Wirth LJ, et al. Lenvatinib versus placebo in radioiodine-refractory thyroid cancer. $\mathrm{N}$ Engl J Med 2015;372:621-30.

6. Cabanillas ME, de Souza JA, Geyer S, et al. Cabozantinib As Salvage Therapy for Patients With Tyrosine Kinase Inhibitor-Refractory Differentiated Thyroid Cancer: Results of a Multicenter Phase II International Thyroid Oncology Group Trial. J Clin Oncol 2017;35:3315-21.

7. Xing M. Molecular pathogenesis and mechanisms of thyroid cancer. Nat Rev Cancer 2013;13:184-99.

8. Xing M, Haugen BR, Schlumberger M. Progress in molecular-based management of differentiated thyroid cancer. Lancet 2013;381:1058-69.

9. Yu XM, Lo CY, Lam AK, et al. Serum vascular endothelial growth factor $\mathrm{C}$ correlates with lymph node metastases and high-risk tumor profiles in papillary thyroid carcinoma. Ann Surg 2008;247:483-9.

10. Salajegheh A, Smith RA, Kasem K, et al. Single nucleotide polymorphisms and mRNA expression of VEGF-A in papillary thyroid carcinoma: potential markers for aggressive phenotypes. Eur J Surg Oncol 2011;37:93-9.

11. Roy S, Narang BK, Rastogi SK, et al. A novel multiple tyrosine-kinase targeted agent to explore the future perspectives of anti-angiogenic therapy for the treatment 
of multiple solid tumors: cabozantinib. Anticancer Agents Med Chem 2015;15:37-47.

12. Markowitz JN, Fancher KM. Cabozantinib: A Multitargeted Oral Tyrosine Kinase Inhibitor. Pharmacotherapy 2018;38:357-69.

13. Ho AL, Grewal RK, Leboeuf R, et al. Selumetinibenhanced radioiodine uptake in advanced thyroid cancer. N Engl J Med 2013;368:623-32.

14. Spano JP, Vano Y, Vignot S, et al. GEMOX regimen in the treatment of metastatic differentiated refractory thyroid carcinoma. Med Oncol 2012;29:1421-8.

15. Haugen BR, Sherman SI. Evolving approaches to patients with advanced differentiated thyroid cancer. Endocr Rev 2013;34:439-55.

16. Kimura ET, Nikiforova MN, Zhu Z, et al. High prevalence of BRAF mutations in thyroid cancer: genetic evidence for constitutive activation of the RET/PTC-RAS-BRAF signaling pathway in papillary thyroid carcinoma. Cancer Res 2003;63:1454-7.

17. Xing M, Alzahrani AS, Carson KA, et al. Association between BRAF V600E mutation and mortality in patients with papillary thyroid cancer. JAMA 2013;309:1493-501.

18. Yarchoan M, LiVolsi VA, Brose MS. BRAF mutation and thyroid cancer recurrence. J Clin Oncol 2015;33:7-8.

19. Nikiforov YE, Nikiforova MN. Molecular genetics and diagnosis of thyroid cancer. Nat Rev Endocrinol 2011;7:569-80.

20. McCain J. The MAPK (ERK) Pathway: Investigational Combinations for the Treatment Of BRAF-Mutated Metastatic Melanoma. P T 2013;38:96-108.

21. Sun C, Wang L, Huang S, et al. Reversible and adaptive resistance to BRAF(V600E) inhibition in melanoma. Nature 2014;508:118-22.

22. Corcoran RB, Ebi H, Turke AB, et al. EGFR-mediated reactivation of MAPK signaling contributes to insensitivity of BRAF mutant colorectal cancers to RAF inhibition with vemurafenib. Cancer Discov 2012;2:227-35.

23. Haarberg HE, Smalley KS. Resistance to Raf inhibition in cancer. Drug Discov Today Technol 2014;11:27-32.

24. Shi H, Moriceau G, Kong X, et al. Melanoma whole-

Cite this article as: Tsoukalas N, Tsapakidis K, Alexandraki KI. The role of palbociclib in thyroid carcinoma with BRAF mutation. Gland Surg 2018;7(Suppl 1):S82-S85. doi: 10.21037/ gs.2018.03.07 exome sequencing identifies (V600E)B-RAF amplificationmediated acquired B-RAF inhibitor resistance. Nat Commun 2012;3:724.

25. Whittaker SR, Theurillat JP, Van Allen E, et al. A genome-scale RNA interference screen implicates NF1 loss in resistance to RAF inhibition. Cancer Discov 2013;3:350-62.

26. Poulikakos PI, Persaud Y, Janakiraman M, et al. RAF inhibitor resistance is mediated by dimerization of aberrantly spliced BRAF(V600E). Nature 2011;480:387-90.

27. Chambard JC, Lefloch R, Pouysségur J, et al. ERK implication in cell cycle regulation. Biochim Biophys Acta 2007;1773:1299-310.

28. Smalley KS, Lioni M, Dalla Palma M, et al. Increased cyclin D1 expression can mediate BRAF inhibitor resistance in BRAF V600E-mutated melanomas. Mol Cancer Ther 2008;7:2876-83.

29. Nathanson KL, Martin AM, Wubbenhorst B, et al. Tumor genetic analyses of patients with metastatic melanoma treated with the BRAF inhibitor dabrafenib (GSK2118436). Clin Cancer Res 2013;19:4868-78.

30. Antonello ZA, Hsu N, Bhasin M, et al. Vemurafenibresistance via de novo RBM genes mutations and chromosome 5 aberrations is overcome by combined therapy with palbociclib in thyroid carcinoma with BRAFV600E. Oncotarget 2017;8:84743-60.

31. Finn RS, Crown JP, Lang I, et al. The cyclin-dependent kinase 4/6 inhibitor palbociclib in combination with letrozole versus letrozole alone as first-line treatment of oestrogen receptor-positive, HER2-negative, advanced breast cancer (PALOMA-1/TRIO-18): a randomised phase 2 study. Lancet Oncol 2015;16:25-35.

32. Finn RS, Martin M, Rugo HS, et al. Palbociclib and letrozole in advanced breast cancer. N Engl J Med 2016;375:1925-36.

33. Balmelli C, Railic N, Siano M, et al. Lenvatinib in Advanced Radioiodine-Refractory Thyroid Cancer - A Retrospective Analysis of the Swiss Lenvatinib Named Patient Program. J Cancer 2018;9:250-5. 